\title{
Keyword Recommendation Methods for Earth Science Data Considering Hierarchical Structure of Vocabularies
}

\author{
Toshiyuki Shimizu \\ Graduate School of Informatics, \\ Kyoto University \\ Kyoto, Japan \\ tshimizu@i.kyoto-u.ac.jp
}

\author{
Joji Kido \\ Graduate School of Informatics, \\ Kyoto University \\ Kyoto, Japan \\ kido.joji.84c@st.kyoto-u.ac.jp
}

\author{
Masatoshi Yoshikawa \\ Graduate School of Informatics, \\ Kyoto University \\ Kyoto, Japan \\ yoshikawa@i.kyoto-u.ac.jp
}

\begin{abstract}
To understand and properly use scientific data, it is important that the metadata, which describes information related to data, contains sufficient information. Keywords are one of the metadata items, and for assigning keywords to the earth science dataset, it is common to select and assign appropriate keywords from a controlled vocabulary with a hierarchical structure. Keyword information plays an important role in dataset search and classification; however, the cost of selecting appropriate keywords from a controlled vocabulary is high, and in many cases, a sufficient number of keywords are not actually assigned. In this study, we focus on keyword recommendations for earth science datasets using the definition sentences given to the keywords in the controlled vocabulary, and propose content-based recommendation methods considering the hierarchical structure in the controlled vocabulary.
\end{abstract}

\section{CCS CONCEPTS}

- Information systems $\rightarrow$ Recommender systems.

\section{KEYWORDS}

keyword recommendation; scientific data; metadata

\section{ACM Reference Format:}

Toshiyuki Shimizu, Joji Kido, and Masatoshi Yoshikawa. 2020. Keyword Recommendation Methods for Earth Science Data Considering Hierarchical Structure of Vocabularies. In Proceedings of the ACM/IEEE Foint Conference on Digital Libraries in 2020 ( $\mathcal{~ C D L ~ ' 2 0 ) , ~ A u g u s t ~ 1 - 5 , ~ 2 0 2 0 , ~ V i r t u a l ~ E v e n t , ~}$ China. ACM, New York, NY, USA, 4 pages. https://doi.org/10.1145/3383583. 3398622

\section{INTRODUCTION}

Due to the development of earth science, there are enormous and diverse earth science data, and the demand for analysis of earth science data has increased significantly. It is important for data users to understand the data and to be able to appropriately search data that they want to use for analysis. In this situation, metadata from earth science datasets play an important role in understanding and searching data. The metadata we assume in this study are given to the earth science data on a dataset basis, and includes the title,

Permission to make digital or hard copies of part or all of this work for personal or classroom use is granted without fee provided that copies are not made or distributed for profit or commercial advantage and that copies bear this notice and the full citation on the first page. Copyrights for third-party components of this work must be honored. For all other uses, contact the owner/author(s).

FCDL '20, August 1-5, 2020, Virtual Event, China

(c) 2020 Copyright held by the owner/author(s).

ACM ISBN 978-1-4503-7585-6/20/08

https://doi.org/10.1145/3383583.3398622 creation date, author, data format, abstract text, and keywords. It is common for such metadata to be described manually by data providers with knowledge of the data.

Keyword information is one of the items of earth science metadata, and it is important that keywords are appropriately given in metadata as they are useful for searching and understanding data. Here, the keywords we assume in this study are not arbitrary keywords but are selected from a controlled vocabulary. In the field of earth science, the GCMD science keywords ${ }^{1}$ vocabulary is commonly used, and this paper assumes the GCMD science keywords are a specific controlled vocabulary. The GCMD science keywords are a controlled vocabulary containing over 3,000 earth sciencespecific keywords, which was developed in the Global Change Master Directory (GCMD) ${ }^{2}$.

However, the cost of selecting appropriate keywords from a controlled vocabulary, which contains many and diverse keywords, is high, and in many cases, keywords are not appropriately assigned [3]. In DIAS (Data Integration Analysis System) ${ }^{3}$ which manages earth science datasets, among 437 datasets annotated in English, 220 have no GCMD science keywords ${ }^{4}$. Additionally, in the case of earth science datasets in GCMD, approximately onefourth of the datasets have fewer than 5 keywords. To improve this situation, we propose assisting the keyword selection by recommending the keywords that seem appropriate for each dataset.

Each keyword in GCMD science keywords has its definition sentences, and in this study, we focus on content-based recommendation using definition sentences. That is, as the metadata for earth science datasets include abstract text for the dataset, we can utilize the similarity between the abstract text of the target dataset and definition sentences of a candidate keyword, and recommend keywords with a high degree of similarity. In this paper, we call a recommendation method based on this approach direct method because it calculates a score for the recommendation (degree of similarity) by directly comparing the target data (abstract text for the target dataset) and the data to be recommended (definition sentences of a candidate keyword).

Recommendation methods based on the concept of collaborative filtering are also possible. If an existing metadata set that contains both abstract text and keywords for each dataset is available, we can recommend keywords assigned to similar existing datasets [6]. In this paper, we call a recommendation method based on this approach indirect method.

\footnotetext{
${ }^{1}$ https://wiki.earthdata.nasa.gov/display/gcmdkey

${ }^{2}$ https://gcmd.nasa.gov/

${ }^{3}$ https://www.diasjp.net/en/

${ }^{4}$ As of August 2019
} 
In keyword recommendation using metadata for earth science datasets, since the indirect method uses a set of existing metadata, the accuracy of keyword recommendations depends on the quality of the existing metadata set. However, since the direct method does not use the existing metadata set, the accuracy of keyword recommendation does not depend on the quality of the existing metadata set, and only depends on the metadata quality of the target dataset. Therefore, when the quality of the existing metadata set is low, the direct method is more effective than the indirect method [3]. We consider it very important to address the situation where the quality of the existing metadata set is low because metadata databases immediately after their creation always face that situation.

In addition, the GCMD science keywords has a hierarchical structure. For example, the keyword "DROPLET GROWTH" is actually on the path "ATMOSPHERE > CLOUDS > CLOUD MICROPHYSICS > DROPLET GROWTH", and each keyword on the path has its definition sentences. Additionally, we observe the definition sentences in the GCMD science keywords are sometimes too short and hard to understand without referring to the definition sentences of the ancestor keywords. We think this nature of the GCMD science keywords is unique, and it is important to develop recommendation methods considering this nature. Therefore, in this study, we propose recommendation methods that consider the keyword hierarchy when performing keyword recommendations based on the direct method.

\section{RELATED WORK}

Keywords are used in various situations, and there are many studies on keyword recommendation [1-3, 5, 6]. Here, we introduce related research on keyword recommendation methods when keywords have a hierarchical structure.

Santos et al. proposed multilabel hierarchical text classification methods [5]. They used the ACM classification scheme as a controlled vocabulary, and built classifiers in each level of the hierarchy. Bi et al. also proposed a multilabel hierarchical classification method which can be used on both tree- and DAG-structured hierarchies [1]. We introduce these studies because we can regard classification as the assignment of keywords. We can categorize the methods in these studies into indirect methods, which is not the focus of this paper.

Contractor et al. developed the system for labeling digital learning content with the learning standards [2]. The learning standards they used can be regarded as controlled vocabularies with a hierarchical structure, and the most granular objects are associated with textual description. Their study is similar to ours because it considers the keyword hierarchy and employs a direct method. However, the nature of the controlled vocabulary used in their study is different from ours as the textual description is stored only in leaf nodes, while all keywords in the GCMD science keywords used in our study have definition sentences.

\section{BASELINE METHOD}

\subsection{Implementation of the Baseline Method}

As described in Section 1, we consider keyword recommendation based on the direct method in this study. The basic concept is to calculate the similarity between the abstract text of the target dataset and definition sentences of each candidate keyword, then recommend keywords with high similarity. Therefore, the method assumes that definition sentences of a keyword have a certain amount of text information for the similarity calculation. From the preliminary experiments, we found that the naive use of definition sentences of the GCMD science keywords often fall into low accuracy recommendations because they are sometimes too short. For example, the definition sentence of the keyword "DROPLET GROWTH" in the GCMD science keywords is just "Rate of droplet growth", and this is too short to make an appropriate recommendation.

Based on this observation, we decided to utilize not only the definition sentences of the keyword but also the definition sentences of the keywords at the upper level on the path in the vocabulary hierarchy. More specifically, for the keyword "DROPLET GROWTH" having a path "ATMOSPHERE > CLOUDS > CLOUD MICROPHYSICS > DROPLET GROWTH", we use the concatenation of the definition sentences of "ATMOSPHERE", "CLOUDS", "CLOUD MICROPHYSICS", and "DROPLET GROWTH”. In this paper, we call these concatenated definition sentences extended definition sentences. We represent the definition sentences of a keyword $k_{j}$ by $D_{j}$, and the extended definition sentences of a keyword $k_{j}$ by $\hat{D}_{j}$. For a keyword $k_{3}$ on a path $k_{1}>k_{2}>k_{3}, \hat{D}_{3}$ is equal to $D_{1}+D_{2}+D_{3}$, where + represents the concatenation.

In this paper, we regard the naive method using extended definition sentences as the baseline method. Here, we represent the abstract text of the target dataset $d_{i}$ by $A_{i}$. The baseline method creates feature vectors for $A_{i}$ and each $\hat{D}_{j}$ using the TF-IDF weighting scheme, and recommends keywords in descending order of cosine similarity between them. For the term frequency (TF), we use the length regularized TF (LRTF) introduced in [4], which is suitable for long queries consisting of more than 5 words. Note that, in our situation, an abstract text of a target dataset is treated as a query, and the number of words in abstract texts is generally more than 5. The inverse document frequency (IDF) is calculated using the standard formula.

\subsection{Baseline Method Problem}

In the baseline method, when the similarity between the definition sentences of keywords at the upper level in the hierarchy and the abstract text of the target dataset is high, keywords with the same upper path tend to be recommended. Figure 1 shows an example of the result of keyword recommendation using the baseline method. In this example, due to the high similarity between the definition sentences of "ATMOSPHERE" or "ATMOSPHERIC RADIATION" and the abstract text of the target dataset, only keywords with the path "ATMOSPHERE > ATMOSPHERIC RADIATION" were recommended. We consider this undesirable because the similarity between the definition sentences of the keyword and the abstract text of the target dataset may be low in such a situation.

\section{PROPOSED METHODS}

We devise the KR (keyword removal) method and the WA (weight adjustment) method to improve the baseline method. Furthermore, the combinations of these methods are also examined. 


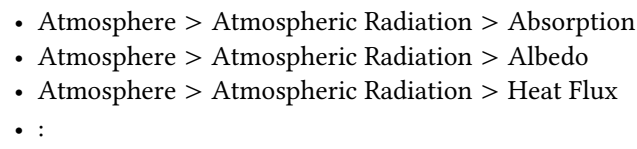

Figure 1: An example of an undesirable recommendation.

\subsection{KR Method}

The KR method is a method that removes the keywords that seem to have low relevance to the target dataset from the candidates of the recommended keywords, and then applies the baseline method. In this paper, we propose the KR-S method and KR-U method, considering two kinds of criteria to remove keywords from recommendation candidates.

4.1.1 KR-S Method. When the problem described in Section 3.2 occurs, it is possible that the similarity between the definition sentences of the keyword and the abstract text of the target dataset is low, though the similarity between the extended definition sentences and the abstract text is high. The low similarity between the definition sentences of the keyword and the abstract text of the target dataset means that the keyword has little relevance to the target dataset; thus, it is not preferable to recommend such keywords.

The KR-S (keyword removal based on similarity) method is a method for filtering out keywords based on the similarity between the definition sentences of the keyword and the abstract text of the target dataset. If the similarity between the definition sentence $D_{j}$ of the keyword $k_{j}$ and the abstract text $A_{i}$ of the target dataset $d_{i}$ is below a certain threshold $s$, the keyword $k_{j}$ is removed from the candidates of recommended keywords. After that, the recommended keywords are ranked by the same process as the baseline method using the extended definition sentences.

4.1.2 KR-U Method. If the cosine similarity between the feature vectors of the definition sentences $D_{j}$ of the keyword $k_{j}$ and the abstract text $A_{i}$ of the target dataset $d_{i}$ is 0 , that is if there is no matching word, it is considered appropriate to remove $k_{j}$ from the candidates of recommended keywords since such keywords seem not to be relevant to the target dataset. Furthermore, even when $D_{j}$ and $A_{i}$ have some matching words, $k_{j}$ should be removed from the candidates if the matching words are common words used in various earth science fields. We assume that the words that appear in the definition sentences of the keywords at the upper level in the hierarchy are common words.

The KR-U (keyword removal based on term uniqueness) method is a method to retain keywords as candidates only when the definition sentences of the keyword and the abstract text of the target dataset matches the words specific to the keyword. Here, we represent the set of words included in $D_{j}$ by $T\left(D_{j}\right)$, the set of words included in $A_{i}$ by $T\left(A_{i}\right)$, and the set of keywords in the upper level of a keyword $k_{j}$ by ancestors $\left(k_{j}\right)=\left\{k_{a(1)}, k_{a(2)} \ldots, k_{a(l)}\right\}$. For a keyword $k_{j}$, if the word set $U=T\left(A_{i}\right) \cap\left(T\left(D_{j}\right)-\left(\bigcup_{n=1}^{l} T\left(D_{a(n)}\right)\right)\right)$ becomes $\phi$, the keyword $k_{j}$ is removed from the candidates of recommended keywords. After that, recommended keywords are ranked by the same process as the baseline method using the extended

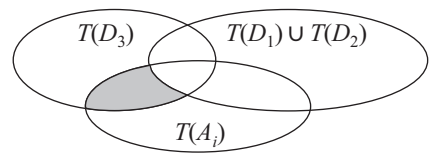

Figure 2: Word set $U$ for a keyword $k_{3}$ on a path $k_{1}>k_{2}>k_{3}$.

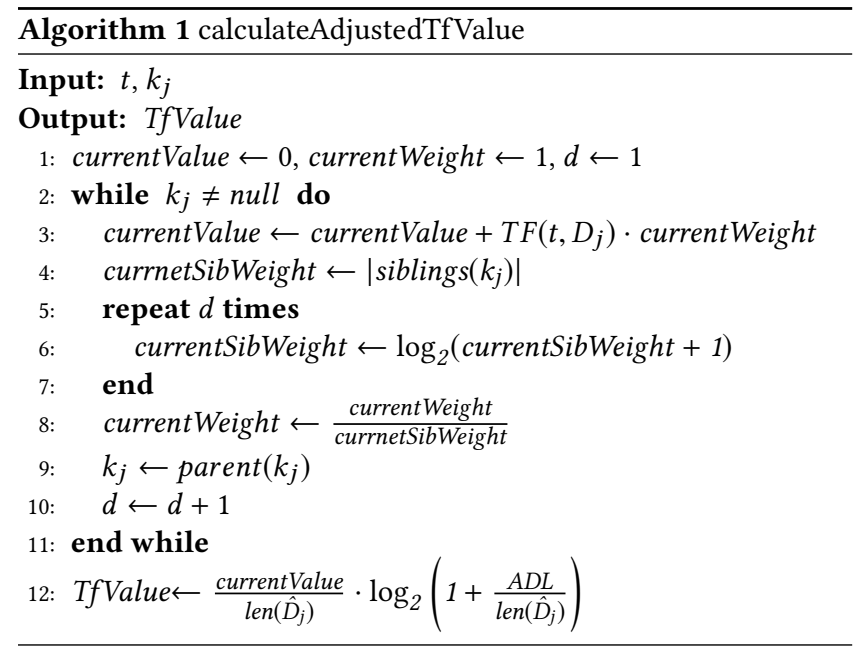

definition sentences. For a keyword $k_{3}$ on a path $k_{1}>k_{2}>k_{3}$, ancestors $\left(k_{3}\right)$ is $\left\{k_{1}, k_{2}\right\}$, and the word set $U$ is represented by the shaded part in the Venn diagram of Figure 2.

\subsection{WA Method}

The importance or weight of words in the extended definition sentences differs depending on the position of the keyword in the hierarchy, and the words appearing at the upper level have lower importance. In addition, when there are many sibling keywords, the weight of the word appearing in the definition sentences of the parent keyword should be reduced to emphasize the difference in the sibling keywords. If the number of sibling keywords is large, the problem described in Section 3.2 is more likely to occur, and we can improve the situation by appropriately adjusting the weight of words in the extended definition sentences depending on the position in which the word appears in the hierarchical structure.

We propose the WA method that adjusts the weight of words in the extended definition sentences when calculating weight using TF-IDF. The WA method adjusts the TF value of TF-IDF to consider the above discussion, and specifically, Algorithm 1 is used to calculate the TF value. Algorithm 1 calculates the adjusted TF value of the word $t$ that appears in the extended definition sentences $\hat{D}_{j}$ of the keyword $k_{j}$. Here, len $\left(\hat{D}_{j}\right)$ is the number of words in the extended definition sentences $\hat{D}_{j}, A D L$ is the average number of words of all extended definition sentences, and $T F\left(t, D_{j}\right)$ is the appearance frequency of word $t$ in $D_{j}$. Additionally, parent $\left(k_{j}\right)$ indicates the parent keyword of $k_{j}$ and $\left|\operatorname{siblings}\left(k_{j}\right)\right|$ indicates the number of sibling keywords of $k_{j}$. 
Table 1: $n h C G$ value of each method

\begin{tabular}{l|l}
\hline Method & $n h C G$ value \\
\hline \hline Baseline & 0.0796 \\
\hline KR-S & 0.0854 \\
\hline KR-U & 0.0815 \\
\hline WA & 0.0809 \\
\hline KR-S \& WA & 0.0800 \\
\hline KR-U \& WA & 0.0807 \\
\hline
\end{tabular}

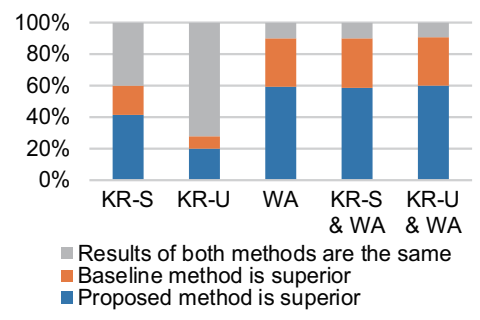

Figure 3: Comparison of each proposed method and baseline method.

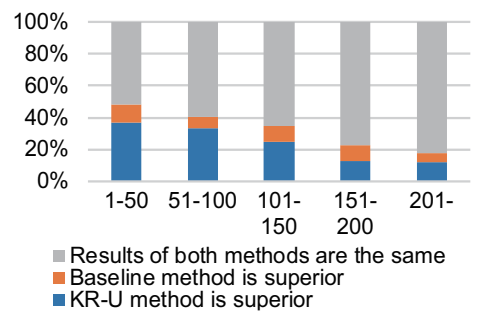

Figure 4: Comparison of the KR-U method and baseline method.

\subsection{Combination of KR and WA Methods}

As a combination of the KR method and the WA method, it is possible to apply the WA method after filtering the candidates for the recommended keywords by the KR method. Since we consider the KR-S method and the KR-U method for the KR method, there are two possible combinations, that is the KR-S \& WA method and the KR-U \& WA method.

\section{EXPERIMENTAL EVALUATION}

We conducted experiments comparing the accuracy of the baseline method and the proposed methods. For our experiment, we used real metadata from earth science datasets in GCMD. Because metadata in the GCMD includes both abstract text and keywords of the GCMD science keywords, we can utilize them for the evaluation by regarding the annotated GCMD science keywords as the set of correct keywords for that dataset. We use 4,690 metadata that are annotated with more than 10 keywords. As the evaluation metrics, we use $n h C G_{D S r e d u c e} @ 10 . n h C G$ (normalized hierarchical cumulated gain) is an evaluation metric that considers the hierarchical structure [3]. For the threshold $s$ used in the KR-S method, we tested different thresholds in the preliminary experiment, and chose 0.09 , which is the value that produced the best results. The threshold in the KR-S \& WA method was also set to 0.09 .

Table 1 shows the average value of $n h C G$ for each method. Additionally, for each of the proposed methods KR-S, KR-U, WA, KR-S \& WA, and KR-U \& WA, we counted the number of datasets in which the proposed method achieved better results than the baseline method in terms of the $n h C G$ value, and observed the proportion. The result is shown in Figure 3.

All of the proposed methods improved the recommendation accuracy compared to the baseline method on average, and overall, the accuracy improved most by the KR-S method. From Figure 3, we observe that the recommendation results of the WA method are different from that of the baseline method for most of the datasets, while the KR-U method affects a smaller number of datasets. We observe little difference between the WA method, the KR-S \& WA method, and the KR-U \& WA method because the keywords removed by the KR methods are unlikely to become recommended keywords in the WA method.

Furthermore, we examined the changes in the results depending on the number of words in the abstract text of the target dataset. We divided the 4,690 metadata into five groups: those with 1-50 words (401 dataset), 51-100 words (893 dataset), 101-150 words (807 dataset), 151-200 words (774 dataset), and more than 200 words
(1,815 dataset). Figure 4 shows a comparison between the KR-U method and the baseline method that yielded particularly interesting results. We observe that the KR-U method is especially effective when the number of words in the abstract text is small. In the direct method, there is a tendency that the larger the number of words in the abstract text of the target dataset, the higher the keyword recommendation accuracy [3]. Therefore, the improvement by the KR-U method when the number of words in the abstract text is small is important.

\section{CONCLUSION}

In this paper, we focused on the GCMD science keywords which is a controlled vocabulary with a hierarchical structure, and proposed keyword recommendation methods considering the hierarchy. First, we introduced a baseline method that uses extended keyword definition sentences, and then proposed some methods that can improve the baseline method. Our experimental evaluation showed that the overall recommendation accuracy of the proposed methods improved compared to the baseline method. Additionally, different improvement tendencies were observed among the proposed methods. Future work includes the development of a more appropriate combination method of the KR method and the WA method, and improvement of the weight adjustment in the WA method. Though we developed the methods considering the unique features of the GCMD science keywords, we are also interested in applying our methods to other domains.

\section{ACKNOWLEDGMENTS}

This work was partially supported by the Data Integration and Analysis System (DIAS) of the Ministry of Education, Culture, Sports, Science and Technology, Japan (MEXT).

\section{REFERENCES}

[1] Wei Bi and James T. Kwok. 2011. Multi-label Classification on Tree- and DAGStructured Hierarchies. In ICML. 17-24.

[2] Danish Contractor, Kashyap Popat, Shajith Ikbal, Sumit Negi, Bikram Sengupta, and Mukesh K. Mohania. 2015. Labeling Educational Content with Academic Learning Standards. In SDM. 136-144.

[3] Youichi Ishida, Toshiyuki Shimizu, and Masatoshi Yoshikawa. 2020. An analysis and comparison of keyword recommendation methods for scientific data. Int. $\mathcal{F}$. on Digital Libraries (2020).

[4] Jiaul H. Paik. 2013. A novel TF-IDF weighting scheme for effective ranking. In SIGIR. 343-352.

[5] António Paulo Santos and Fátima Rodrigues. 2009. Multi-label hierarchical text classification using the ACM taxonomy. In EPIA. 553-564.

[6] Suppawong Tuarob, Line C. Pouchard, and C. Lee Giles. 2013. Automatic tag recommendation for metadata annotation using probabilistic topic modeling. In FCDL. 239-248. 